\title{
Comparing different dynamic stall models
}

\author{
J.G. Holierhoek \\ J.B. de Vaal $^{1}$ \\ A.H. van Zuijlen ${ }^{1}$ \\ H. Bijl ${ }^{1}$ \\ ${ }^{1}$ Aerospace Engineering, Delft University of Technology
}

Published online in Wiley Online Library (wileyonlinelibrary.com). DOI: 10.1002/we.548, Wind Energ. (2012) 


\title{
RESEARCH ARTICLE
}

\section{Comparing different dynamic stall models}

\author{
J. G. Holierhoek ${ }^{1}$, J. B. de Vaal' ${ }^{2}$, A. H. van Zuijlen² and H. Bijl² \\ 1 Unit Wind Energy, Energy research Centre of the Netherlands, PO Box 1, 1755 ZG Petten, The Netherlands \\ 2 Aerospace Engineering, Delft University of Technology, Kluyverweg 1, 2629 HS Delft, The Netherlands
}

\begin{abstract}
The dynamic stall phenomenon and its importance for load calculations and aeroelastic simulations is well known. Different models exist to model the effect of dynamic stall; however, a systematic comparison is still lacking. To investigate if one is performing better than another, three models are used to simulate the Ohio State University measurements and a set of data from the National Aeronautics and Space Administration Ames experimental study of dynamic stall and compare results. These measurements were at conditions and for aerofoils that are typical for wind turbines, and the results are publicly available. The three selected dynamic stall models are the ONERA model, the Beddoes-Leishman model and the Snel model. The simulations show that there are still significant differences between measurements and models and that none of the models is significantly better in all cases than the other models. Especially in the deep stall regime, the accuracy of each of the dynamic stall models is limited. Copyright @ 2012 John Wiley \& Sons, Ltd.
\end{abstract}

\section{KEYWORDS}

dynamic stall; unsteady aerodynamics; aeroelasticity

\section{Correspondence}

J. G. Holierhoek, Unit Wind Energy, Energy research Centre of the Netherlands, PO Box 1, 1755 ZG Petten, The Netherlands.

E-mail: holierhoek@ecn.nl

Received 17 January 2011; Revised 21 October 2011; Accepted 17 November 2011

\section{INTRODUCTION}

Recent years have seen a significant growth in the size and investment costs of wind turbines. Along with this growth in the market, there is an equally growing demand for more reliable design methods. This is especially the case for off-shore wind turbines, where reliability of the turbine and its components are among the most important challenges faced by designers. However, uncertainty in design calculations restricts the confidence levels to which turbines can be designed reliably today. At present, the uncertainty in design calculations is $10 \%$ to $20 \%$ for performance prediction and $30 \%$ for dynamic load prediction. ${ }^{1}$ To enable more reliable calculations, both the structural dynamics as well as the aerodynamics will have to be improved. To enable the use of general purpose structural codes (e.g. multi body codes) for wind turbine simulations, the Energy research Centre of the Netherlands (ECN) is developing the ECN-Aero-Module, ${ }^{2}$ whereby different aerodynamic models can be coupled to the structural codes.

One of the reasons for the large uncertainty in the dynamic load prediction for wind turbines is the unsteady (and often unpredictable) nature of the environment in which they typically operate. The unsteady flow conditions in which wind turbines operate complicate the process of determining accurate aerodynamic force coefficients. The wind field encountered by a wind turbine deviates from uniform inflow because of local atmospheric and topographical influences such as wind shear, turbulence and wind gusts. Also, yaw misalignment, rotor tilt, deformations and the presence of the support structure cause each section of a rotor blade to experience unsteady flow.

In combination with the unsteady flow environment, high aerodynamic angles of attack where the flow is stalled are often, sometimes unavoidably or intentionally, encountered on wind turbine blades. It is evident that stall plays a very important role in a wind turbine's normal operation.

According to Leishman, ${ }^{3}$ dynamic stall will occur on any aerofoil or other lifting surface when it is subjected to timedependent pitching, plunging or vertical translation, or other types of motion that take the effective angle of attack above its normal steady stall angle. For dynamic stall, the physics of the flow separation and stall development differs fundamentally from the stall mechanisms observed for the same aerofoil under static (quasi-steady) conditions. ${ }^{4}$ 
Stated simply, the effect of dynamic stall is a delay in stall onset: stall occurs at a higher angle of attack than for the static stall case. A strong vortex is formed at the leading edge that separates and is convected along the upper surface of the aerofoil. This event begins with a rapid increase in lift and ends with full flow separation and catastrophic loss of lift as the vortex disturbance is convected past the trailing edge of the aerofoil. ${ }^{4,5}$ This behaviour can produce hysteresis loops in the force coefficients (see Figure 1), producing cyclic pressure loadings that are not predicted by conventional lift and drag data obtained at steady angles of attack. ${ }^{6}$

Experimental results have confirmed that aerodynamic load variations, characteristic of dynamic stall, occur on wind turbine blades and that dynamic stall modelling can help to more accurately define operational and performance boundaries for wind turbines. ${ }^{8,9}$ The accurate prediction of dynamic stall onset (leading edge vortex separation) and the subsequent effects on blade loads and performance are, however, very difficult. ${ }^{3}$

The experimental study of dynamic stall relies heavily on wind tunnel tests of oscillating two-dimensional aerofoils, where the majority of documented experimental results are for pitching aerofoils and only limited amounts of data for e.g. plunging oscillations. ${ }^{3}$ It is clear that the effect of dynamic stall should be incorporated in wind turbine simulations. However, different dynamic stall models exist, and a comparison of different models to identical measurements has not yet been performed for representative wind turbine conditions. Comparisons do exist for representative helicopter conditions, ${ }^{10,11}$ where it has become clear that the two-dimensional models are very important for the representation of dynamic stall but that there is still many needs for further improvements. For wind turbine conditions, however, a comparison has not yet been made; therefore, it is not clear if one model is better than the other, in general or for certain cases. To enable comparison, different models should be compared with one single set of measurements. Of course, each of the different available dynamic stall models has been validated using a set of measurements, but often different sets are used for different models. This inhibits direct comparison. Therefore, in this paper, one set of measurements that represent conditions of wind turbine blades will be used to enable direct comparison of different models.

At Ohio State University (OSU) measurements were performed on pitching wind turbine aerofoils. The publicly available OSU measurements ${ }^{7}$ are representative for modern multimegawatt wind turbine conditions towards the tip of the blade, when looking at the reduced frequency. The Reynolds numbers of around 1.2 million are also representative for wind turbines, which makes the OSU measurements very useful to compare different dynamic stall models with these measurements. The measurements were performed using three different aerofoils: the NACA 4415, the S809 and the LS(1)-0421MOD. ${ }^{12}$ The results from these measurements can serve as a reference to compare the accuracy of different dynamic stall models. For example, consider Figure 1, where the measured lift coefficient for the NACA 4415 aerofoil is shown. ${ }^{7}$ In addition to steady measurements, measurements for the aerofoil oscillating (pitching) about the quarter chord point at reduced frequencies of $k=0.023, k=0.045$ and $k=0.069$ are shown, with $k=\omega c / 2 V$. It is clear from this figure that the reduced frequency $k$ of the pitch oscillations has a significant influence on the measured lift.

As one of the dynamic stall models used was developed using the OSU measurements and as it is known that measurements at different tunnels will result in different results, another set of measurements has been used for the comparison. For this additional comparison, measurements from National Aeronautics and Space Administration (NASA) Ames experimental study of dynamic stall ${ }^{13}$ have been used. A set of measurements was chosen for which the steady and unsteady data were available for the same Mach number and Reynolds number, for which the reduced frequencies were in the range of the OSU measurements and the Mach and Reynolds numbers were the lowest to compare better with wind turbine conditions. The most appropriate measurements turned out to be the measurements for the Vertol VR-7 aerofoil where data are available for a Reynolds number of approximately 2.6 million and reduced frequencies of $k=0.01, k=0.025$ and $k=0.051$.

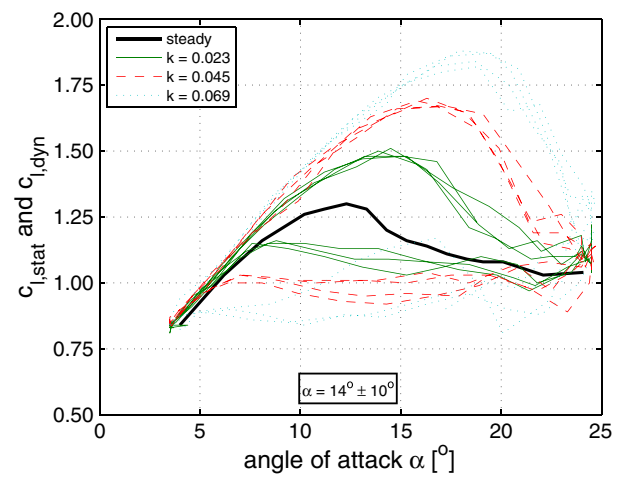

Figure 1. Comparison between the steady and dynamic lift coefficients at different reduced frequencies for the NACA 4415 aerofoil, as measured at Ohio State University. ${ }^{7}$ 
The dynamic stall phenomena do not only influence the size of the loads; it also has significant effect on aeroelastic damping. It is clear that a dynamic stall model has to be included in the tools that are used for aeroelastic load calculations of wind turbines; therefore, the ECN-Aero-Module ${ }^{2}$ should also include at least one dynamic stall model. To investigate which dynamic stall model performs best, three different dynamic stall models have been selected for the comparison, models that do not require many aerofoil specific coefficients and which are often used in wind turbine simulations. These three are ONERA ${ }^{14-17}$, Beddoes-Leishman ${ }^{18-20}$ and Snel. ${ }^{21}$ More details on these three different dynamic stall models will be given in the next section. In Section 3, the results of the comparison between these different models and the OSU and NASA Ames measurements will be discussed. The final section will show the conclusions.

\section{SEMI-EMPIRICAL DYNAMIC STALL MODELLING}

Dynamic stall is currently modelled by computationally efficient engineering models that have originally been developed for helicopter applications and are adapted for wind turbine use. ${ }^{3}$ However, in recent years, focus has shifted to models specifically aimed for use in wind turbine aerodynamic analysis. ${ }^{22}$

Of the dynamic stall models currently in use, the majority are so-called semi-empirical models. These models contain representations of the essential physics using sets of linear and non-linear equations to model the lift, drag and pitching moment coefficients of aerofoils in dynamic conditions. ${ }^{3}$ These equations may require several empirically determined coefficients that have to be deduced from unsteady aerofoil measurements.

In the calculation of the coefficients, many complications occur for dynamic stall models. First, the majority of experimental results for dynamic stall that are available were obtained for aerofoils (and at Reynolds and Mach numbers) that are more applicable for helicopters rather than wind turbines. Second, although the qualitative features of dynamic stall have been found to be the same for a wide range of aerofoils and operating conditions, quantitative behaviour of the aerodynamic coefficients do show subtle variations, especially for different aerofoil shapes, making accurate prediction difficult. ${ }^{3}$ Lastly, even when a dynamic stall model has been tuned to accurately predict certain wind tunnel measurements, these are still not the conditions to which it will be exposed in a real wind turbine operating environment. Dynamic stall models can, therefore, only be used with confidence for situations in which their performance has been verified and validated against experimental data.

In this paper, three different models will be compared with the OSU measurement data, and the results will be compared with each other. These three different models will be discussed next: ONERA, Beddoes-Leishman and Snel.

\subsection{ONERA method}

The ONERA semi-empirical model describes the unsteady aerofoil behaviour by means of a set of non-linear differential equations. A first-order linear differential equation describes the inviscid (attached flow) aerodynamic contribution, and a second-order differential equation describes the non-linear viscous effects associated with stall. The model was originally developed by Tran and Petot ${ }^{14,15,23}$ and further described by McAlister et al. ${ }^{16}$ for rotorcraft applications. Subsequently, modifications that improved the stability of the method were suggested by Peters. ${ }^{17}$ The model was further revised by Petot in $1997 ;{ }^{24}$ however, these corrections are not included in the implemented model. The coefficients that are required for the method's modelling equations are determined by parameter identification from experimental measurements on oscillating aerofoils. A drawback of this method is the large number of empirical coefficients that need to be determined from unsteady experiments. However, good prediction of the actual results seems possible. ${ }^{3}$ Another draw back for wind turbines is that the model is derived on the basis of a linearisation where it is assumed that changes in angle of attack are small and that there is a small difference between the static and dynamic lift coefficients, which is not always the case for wind turbines.

The ONERA model consists of the following two empirically derived differential equations from which the total dynamic lift coefficient is calculated: ${ }^{25}$

$$
\dot{c}_{l, 1}+\lambda_{L} c_{l, 1}=\lambda_{L} c_{l, \mathrm{pot}}+\left(\lambda_{L} s_{L}+\sigma_{L}\right) \dot{\alpha}+s_{L} \ddot{\alpha}
$$

and

$$
\ddot{c}_{l, 2}+a_{L} \dot{c}_{l, 2}+r_{L} c_{l, 2}=-\left(r_{L} \Delta c_{l}+e_{L} \dot{\Delta} c_{l}\right)
$$

with the dynamic lift coefficient equal to the sum:

$$
c_{l, \mathrm{dyn}}=c_{l, 1}+c_{l, 2}
$$

In these equations, the notation $\left({ }^{*}\right)=\mathrm{d} / \mathrm{d} s$ is used to denote differentiation with respect to dimensionless time $s=(2 U / c) t$. The notations are further explained at the end of this paper. In the first-order differential equation (1), the coefficients $\lambda_{L}$, 
$s_{L}$ and $\sigma_{L}$ are functions of the angle of attack and have to be determined from unsteady experimental data. ${ }^{25}$ However, when such unsteady data are not available, which will often be the case, the values for a flat plate may be used. In the calculations discussed in Section 3, no specific aerofoil data have been used; therefore, the flat plate data has been used instead.

For the second-order equation (2), a lift coefficient difference parameter, representing the loss in lift due to flow separation, and its derivative are introduced:

$$
\Delta c_{l}=c_{l, \mathrm{pot}}-c_{l, \mathrm{stat}}
$$

The aerofoil dependent coefficients $a_{L}, r_{L}$ and $e_{L}$ all depend on the loss in lift $\Delta c_{l}$ and are defined as follows:

$$
\begin{gathered}
r_{L}=\left[r_{0}+r_{2}\left(\Delta c_{l}\right)^{2}\right]^{2} \\
a_{L}=a_{0}+a_{2}\left(\Delta c_{l}\right)^{2}
\end{gathered}
$$

and

$$
e_{L}=e_{2}\left(\Delta c_{l}\right)^{2}
$$

The values for the coefficients $a_{0}, a_{2}, r_{0}, r_{2}$ and $e_{2}$ have to be determined for each aerofoil from wind tunnel measurements; flat plate measurements cannot be used. If values for a particular aerofoil are not available, the values for the so-called mean aerofoil can be used instead, ${ }^{26}$ although the prediction is then less accurate. In the calculations discussed in Section 3, the values for the mean aerofoil have been used to model the dynamic stall phenomena.

Because it is defined as a set of differential equations, the form of the ONERA model easily lends itself to be solved together with the equations of motion of a dynamic system. For this reason, in addition to its acceptable prediction of unsteady aerodynamic loads, it is often the method of choice for investigating unsteady effects for wind turbine applications. ${ }^{8,27,28}$ For the comparison discussed in this paper, a second-order accurate backward difference approximation has been used to simulate the conditions as measured in the OSU experiments.

\subsection{Beddoes-Leishman method}

The Beddoes-Leishman method ${ }^{18,19}$ is a dynamic stall model where the emphasis is on a more complete physical representation of the overall unsteady aerodynamic problem but still in a form that keeps the complexity of analysis down to minimise computational cost. ${ }^{3}$ In this way, it attempts to overcome the limitations of other models where a large number of empirically determined coefficients limit the applicability of the method whenever the coefficients cannot be determined for exactly the appropriate flow environment. The model is based on the original work of Beddoes ${ }^{29}$ and has since also been adapted specifically for the class of aerofoils used in wind turbines. ${ }^{20}$

The Beddoes-Leishman model essentially consists of four subsystems: ${ }^{3}$

1. An attached flow model for the unsteady (linear) aerodynamic forces based on Duhamel superposition.

2. A separated flow model for the non-linear aerodynamic forces.

3. A dynamic stall onset model.

4. A dynamic stall model for the vortex induced aerodynamic forces.

These four submodels are connected in an open loop system, where output from one model serves as input for the next. This gives a clear indication of the effect of each submodel on the overall result. The modular approach also serves as inspiration or starting point for other models ${ }^{22,30}$ that use (modified) submodels as building blocks for methods on the basis of the same underlying physics.

Similar to the ONERA method, the Beddoes-Leishman model can be formulated as a set of ordinary differential equations, which is useful for analysis purposes. The main advantage of the Beddoes-Leishman method in comparison with for instance the ONERA method is that it uses relatively few empirically determined coefficients of which all but four can be derived from steady aerofoil data. ${ }^{3}$

Although originally conceived for the analysis of dynamic stall in rotorcraft, where the flow conditions and type of aerofoils are distinctly different from wind turbine applications, the model has been adapted to perform well for wind turbine specific aerofoils even in/near stalled conditions ${ }^{31}$ and is used as dynamic stall model ${ }^{32}$ in wind turbine analysis codes such as Aeroforce. ${ }^{33}$ The Beddoes-Leishman model is the only model of the three discussed dynamic stall models that takes flow separation explicitly into account. 


\subsection{Snel method}

The non-linear, second-order dynamic stall model developed by H. Snel ${ }^{21}$ is an example of a modern semi-empirical engineering model used to include dynamic stall effects in aeroelastic response codes for wind turbines. This is a model that uses no aerofoil specific parameters in its modelling equations but still can predict dynamic stall with the same accuracy as models that do require such input. This characteristic makes Snel's dynamic stall model desirable for application in an aeroelastic design code where numerous aerofoil profiles need to be evaluated, negating the need for any parameter identification from dynamic wind tunnel tests.

On the basis of the observed behaviour of the dynamic lift coefficient, Truong ${ }^{34}$ proposed that the difference between dynamic and steady $c_{l}$ can be written as the sum of two parts, the first part describing the forcing frequency response and the second part describing the higher frequency dynamics of a self-excited nature. The most common dynamic stall models only describe the first part. The Snel model will follow the idea of Truong but with the difference that the first part will also be expressed in terms of $\Delta c_{l}$ or differences with the steady state $c_{l}$. Thus, the dynamic lift coefficient will be written as follows:

$$
c_{l, \mathrm{dyn}}=c_{l, \text { steady }}+\Delta c_{l 1}+\Delta c_{l 2}
$$

For the first-order correction $\Delta c_{l 1}$, Truong makes use of the Beddoes-Leishman model. Snel's model however, uses the SIMPLE model ${ }^{35}$ as point of departure for the modelling of $\Delta c_{l 1}$, using a first-order differential equation of the form

$$
c f_{11} \Delta \dot{c}_{l 1}+c f_{10} \Delta c_{l 1}=f t_{1}
$$

In this manner, $\Delta c_{l 1}$ will follow the frequency of the forcing term, $f t_{1}$. To cast the equation in non-dimensional form, the coefficient of the derivative term, $c f_{11}$, is taken to be equal to the time constant usually considered in dynamic stall, namely $\tau=c / 2 U$, where $c$ is the aerofoil chord length and $U$ the effective wind speed. The forcing term will be based on a derived profile specific quantity, namely the behaviour of $\Delta c_{l \text {,pot }}$

$$
f t_{1}=\tau \Delta \dot{c_{l, p o t}}
$$

where $\Delta c_{l \text {,pot }}$ is defined as the difference between the potential flow lift coefficient and the profile steady lift coefficient:

$$
\Delta c_{l, \mathrm{pot}}=c_{l, \mathrm{pot}}-c_{l, \text { steady }}=2 \pi \sin \left(\alpha-\alpha_{0}\right)-c_{l, \text { steady }}
$$

The coefficient $c f_{10}$ may be seen as the stiffness of a spring trying to pull $\Delta c_{l 1}$ to its steady value of zero, when there is no forcing. As in the SIMPLE model, ${ }^{35}$ the spring is stiffer for negative $\dot{\alpha}$ and also increases its stiffness with increasing $\alpha$. This behaviour is obtained by choosing

$$
c f_{10}= \begin{cases}\frac{1+0.5 \Delta c_{l, \mathrm{pot}}}{8(1+60 \tau \dot{\alpha})} & \text { if } \dot{\alpha} c_{l, \mathrm{pot}} \leq 0 \\ \frac{1+0.5 \Delta c_{l, \mathrm{pot}}}{8(1+80 \tau \dot{\alpha})} & \text { if } \dot{\alpha} c_{l, \mathrm{pot}}>0\end{cases}
$$

The differential equation for $\Delta c_{l 2}$ must be of second order since it should introduce the higher frequency dynamics, which should resemble an eigenfrequency of the equation. The general form of the equation may be written as

$$
\tau^{2} \Delta \ddot{c}_{l 2}+c f_{21} \dot{\Delta} c_{l 2}+c f_{20} \Delta c_{l 2}=f t_{2}
$$

The following coefficients were chosen: ${ }^{21}$

$$
c f_{20}=k_{s}^{2}\left[1+3\left(\Delta c_{l 2}\right)^{2}\right]\left[1+3 \dot{\alpha}^{2}\right]
$$

and

$$
c f_{21}= \begin{cases}60 \tau k_{s}\left[-0.01\left(\Delta c_{l, \text { pot }}-0.5\right)+2\left(\Delta c_{l 2}\right)^{2}\right] & \text { if } \dot{\alpha}>0 \\ 2 \tau k_{s} & \text { if } \dot{\alpha} \leq 0\end{cases}
$$

In these equations, $k_{s}$ is the reduced frequency of vortex shedding in a uniform flow, or Strouhal frequency, which is given a value of $0.2 .^{34}$

Lastly, the forcing term is made up of $\Delta c_{l \text {,pot }}$ and its time derivative in the following form:

$$
f t_{2}=0.12\left(-0.15 \Delta c_{l, \text { pot }}+0.05 \Delta \dot{c}_{l, \text { pot }}\right)
$$

To simulate the conditions as measured in the OSU experiments, a second-order accurate backward difference approximation has been used. 


\section{COMPARISON TO THE EXPERIMENTS}

The three dynamic stall models (ONERA, Beddoes-Leishman and Snel), discussed in the previous section, have been used to run simulations representing the OSU measurement conditions. Of course, each of these models has been validated before; however, by comparing each one to the same set of measurements, their accuracy can be compared. First, the measurements are further described, followed by a short discussion on the model settings. Then the lift hysteresis parameters are compared, followed by the differences between average measured data and simulations. The results will be shown for several representative conditions (different aerofoils, reduced frequencies, mean angles of attack, amplitudes).

\subsection{Unsteady measurements}

As described in Section 1, the OSU measurements are representative for the current multimegawatt wind turbines. However, it should be noted that the first draw back is the fact that the Snel model has been validated using this set of measurements; therefore, it is not a completely independent set of data. A second drawback is the fact that it concerns a pitching aerofoil, whereas a plunging aerofoil would be a significantly better representation for the dynamic stall effects on a wind turbine. A third drawback of these measurements is that no attempt was made during the measurements to calibrate the wind tunnel for the unsteady oscillating model conditions; the steady state tunnel calibration was used to set flow conditions while the model was stationary at its mean angle of attack. ${ }^{7,36,37}$ However, the reduced frequencies, Reynolds numbers and the aerofoils had been specifically chosen to represent wind turbines. This, combined with their public availability, makes it into a relatively good set to be used for the comparison.

As was already mentioned in Section 1 , the measurements were performed on three different aerofoils: the NACA 4415, the S809 and the LS(1)-0421MOD ${ }^{12}$ and each for three different reduced frequencies: $k \approx 0.02, k \approx 0.04$ and $k \approx 0.065$. The three dynamic stall models have been compared for each aerofoil and for each measured reduced frequency.

As the Snel model was derived on the basis of the OSU measurements, for a more fair comparison, at least one other data set has to be used. A set from the NASA Ames measurements ${ }^{13}$ was therefore used, although this set is less representative for wind turbines. By selecting data that were available for a relatively low Reynolds number and ranges of the reduced frequencies that were comparable to the OSU data, with an amplitude of around $10^{\circ}$ and a mean angle of attack of around $14^{\circ}$, this has resulted in an acceptable additional set to be used in the comparison. The data set concerns the helicopter Vertol VR-7 aerofoil at a Reynolds number of around 4 million, a mean angle of attack of around $15^{\circ}$ and an amplitude of approximately $10^{\circ}$. Please note that for the larger mean angles of attack, only the OSU measurements have been used in the comparison. The results are summarised below.

\subsection{Model settings}

To summarise the model settings, the parameters for the Beddoes-Leishman and ONERA models are shown in Table I. For the Beddoes-Leishman model, parameter-setting-based values in Leishman and Beddoes ${ }^{18,19}$ have been used. For the ONERA model, the values for the flat plate have been used for the first-order equation and the values for a mean aerofoil have been used for the second-order equation. For the Snel model, both the first-order and second-order models have been used in the comparison.

Table I. Parameters used for the Beddoes-Leishman dynamic stall model (left) and ONERA model (right)

\begin{tabular}{lcccc}
\hline \multicolumn{2}{c}{ Beddoes-Leishman } & & \multicolumn{2}{c}{ ONERA } \\
\cline { 1 - 1 } \cline { 5 - 5 } Parameter & Value & & Parameter & Value \\
\hline$a$ & $340.3 \mathrm{~m} \mathrm{~s}^{-1}$ & & $\lambda_{L}$ & 0.17 \\
$c$ & $0.457 \mathrm{~m}$ & & $S_{L}$ & $\pi$ \\
$A_{1}$ & 0.3 & & $\sigma_{L}$ & 0.3 \\
$A_{2}$ & 0.7 & & $a_{0}$ & 0.3 \\
$b_{1}$ & 0.14 & & $a_{2}$ & 0.2 \\
$b_{2}$ & 0.53 & & $e_{2}$ & -2.86 \\
$K_{\alpha}$ & 0.75 & & $r_{0}$ & 0.2 \\
$T_{1}$ & $c / a$ & & & \\
$T_{p}$ & 2.5 & & & \\
$T_{f}$ & 3.0 & & & \\
$T_{v}$ & 6.0 & & & \\
\hline
\end{tabular}


The dynamic stall models are all executed with time step sizes determined by modelling accuracy considerations. These time steps are much smaller than the measurement intervals in the unsteady wind tunnel tests. Furthermore, just as in the wind tunnel test, the time span of the modelling runs is set such that at least three pitch oscillations of the modelled aerofoil are completed. Next, one full oscillation (consisting of an upstroke and a downstroke) is extracted from the modelled results, for comparison with the averaged experimental values. The dynamic lift coefficient at identical angle of attack intervals as for the averaged measured values is interpolated from the modelled oscillation cycle.

\subsection{Comparison of the lift hysteresis parameter}

One of the methods of comparison between the measured and modelled dynamic lift curves is to compare the lift hysteresis parameter. The lift hysteresis parameter is defined as the difference in lift coefficient between the two points in a cycle when the aerofoil passes through the angle of initial maximum lift (which is always on the upstroke of a cycle). ${ }^{12}$ These values are indicative of the effects oscillation has on the lift coefficient.

In Figure 2(a)-(d), the lift hysteresis parameters for the four aerofoils are plotted against the reduced frequency. These concern measurements and simulations for an average angle of attack of $14^{\circ}$ and an amplitude of $10^{\circ}$. These figures show that there is a strong relationship between the lift hysteresis and the frequency of oscillation-the hysteresis parameter increases with increasing reduced frequency. This trend is observed in all measurements and for all three dynamic stall models.

For the NACA 4415 (Figure 2(a)), the LS(1)-0421MOD (Figure 2(c)) and the Vertol VR-7 (Figure 2(d)), the trends observed are very similar in both the measured and calculated model values, with for the first two aerofoils generally only small differences between the models and the measurements. For the Vertol, the differences are significantly larger. However, for the S809 (Figure 2(b)), the situation is different. In the measurements, there is a larger increase in the hysteresis parameter between the intermediate and highest reduced frequencies than there is between the lowest and intermediate reduced frequencies. This is not captured by any of the dynamic stall models; all models predict a trend similar to the trends observed for the NACA 4415 and LS(1)-0421MOD aerofoils. As stated by Sheng and Galbraith, ${ }^{38}$ the onset of dynamic stall is rather complicated for the S809 aerofoil and difficult to predict using a dynamic stall model.

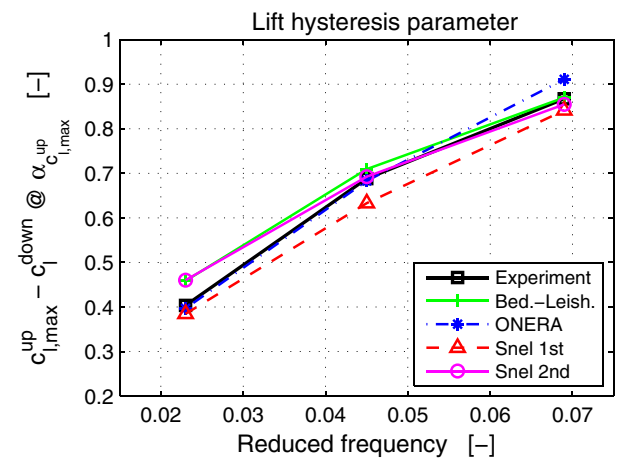

(a) NACA 4415

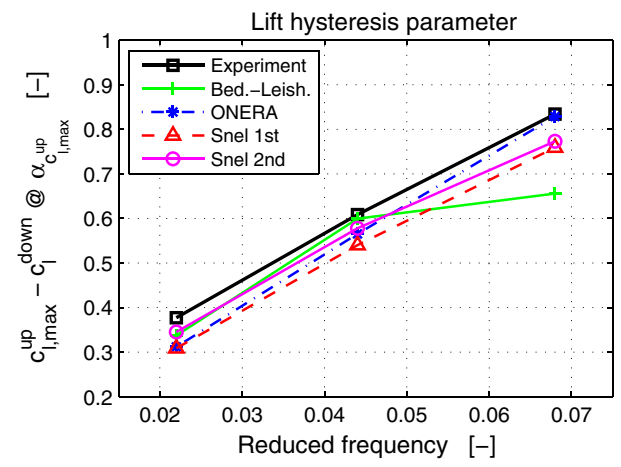

(c) LS(1)-0421MOD

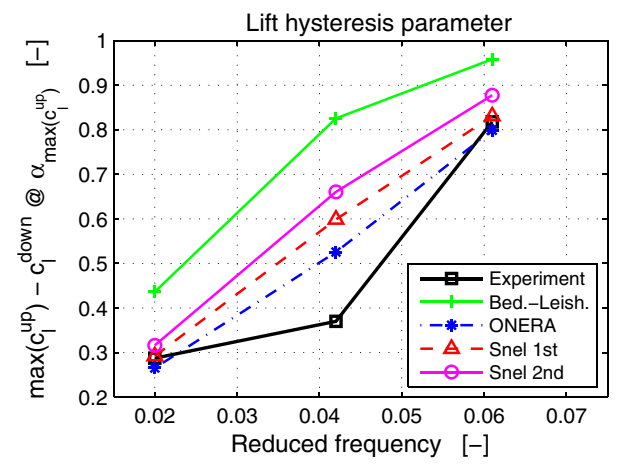

(b) $\$ 809$

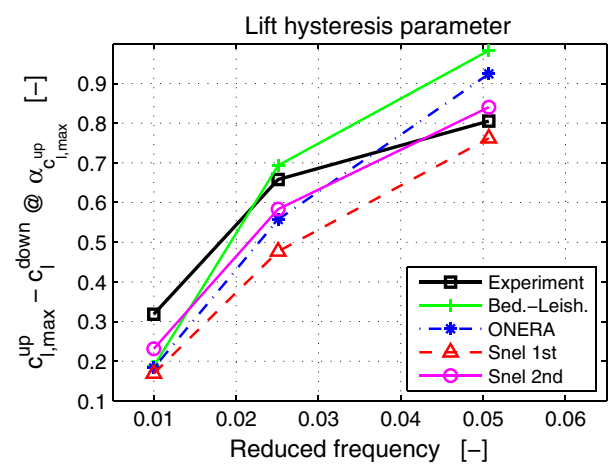

(d) Vertol VR-7

Figure 2. The modelled and measured lift hysteresis parameter for all four aerofoils over the range of reduced frequencies and for Reynolds number approximately $1.25 \times 10^{6}$ for the first three and $4 \times 10^{6}$ for the Vertol VR-7. 
Furthermore, there are substantial differences between the predictions from the various dynamic stall models for the S809 aerofoil and the Vertol VR-7 aerofoil, contrary to the cases for the other two aerofoils. The difference in the hysteresis parameter between the measured and modelled values becomes up to four times greater than for the other two aerofoils. This result might serve as motivation to take differences between aerofoils into account more explicitly since it seems to have an effect on certain predicted results.

Although this test gives no indication of the accuracy of the models throughout the oscillation, it does show that the overall hysteresis effect is captured quite accurately, especially for the NACA 4415 and LS(1)-0421MOD.

Next, the maximum difference in lift coefficient between the upstroke and downstroke for any angle of attack is calculated, with the results shown in Figure 3. For the NACA 4415, LS(1)-0421MOD and Vertol VR-7, nearly identical results as for the lift hysteresis parameter are obtained. But for the S809, the model results remain nearly unchanged, whereas there is a large difference for the measured results. This indicates that for the S809, the maximum difference between the upstroke and downstroke does not occur at the angle of attack for maximum lift coefficient, whereas the dynamic stall models do predict the maximum to occur at the angle of attack for maximum lift coefficient. And for the other three considered aerofoils, this is the case, indicating a significant difference in the behaviour of the S809 aerofoil compared with the NACA, LS(1) and Vertol aerofoils.

There is a strong relationship between increasing pitch oscillation frequency and the magnitude of the stall hysteresis, which the models are predicting correctly. With respect to the location of the maximum difference between an upstroke and a downstroke, it is found to be at or very near the angle for maximum lift. The measurements show that the S809 is the only aerofoil of the four that does not follow this trend.

\subsection{Direct comparison of models to averaged unsteady data}

The first and most evident way of comparing the results is to plot figures showing both the measured and modelled dynamic lift coefficient polars. From these figures, a good first impression of the general trends predicted by the dynamic stall models is obtained. To enable a comparison between different models, the $L_{2}$-norm can be used to give an indication of the difference between the measured and calculated results.

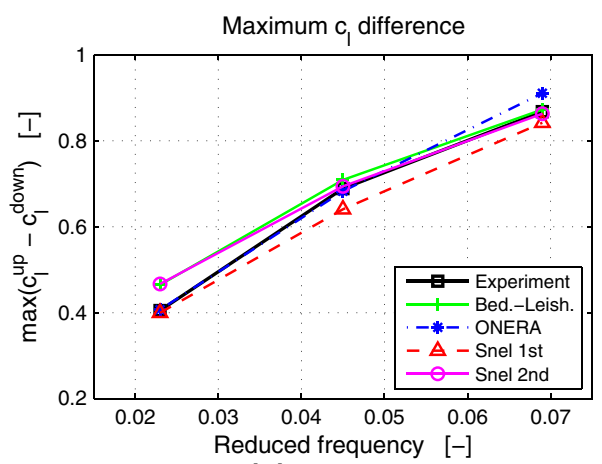

(a) NACA 4415

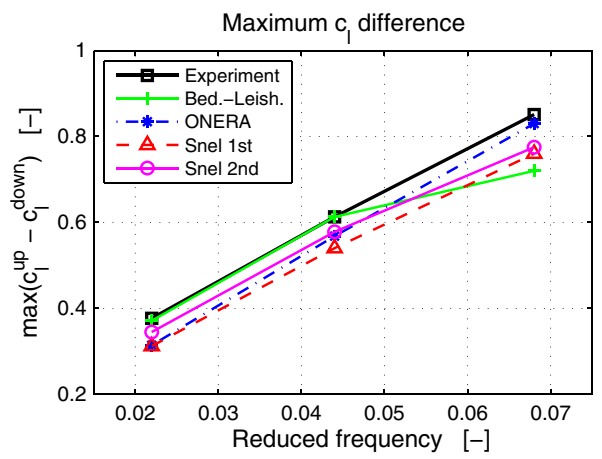

(c) LS(1)-0421MOD

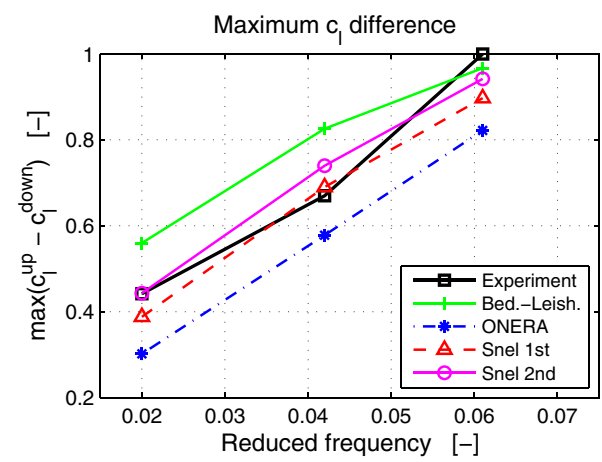

(b) $\$ 809$

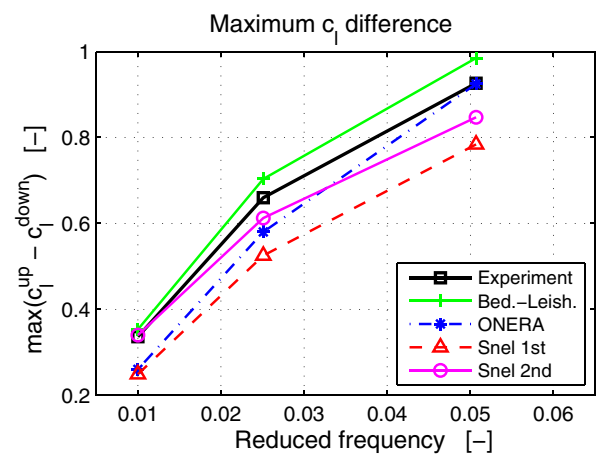

(d) Vertol VR-7

Figure 3. The modelled and measured maximum difference in lift coefficient between the upstroke and downstroke for all four aerofoils over the range of reduced frequencies and for Reynolds number approximately $1.25 \times 10^{6}$ for the first three and $4 \times 10^{6}$ for the Vertol VR-7. 


\section{$L_{2}$ norm of the error}

The $L_{2}$ norm is defined as

$$
\|f(x)\|_{L_{2}}=\sqrt{\int_{a}^{b}|f(x)|^{2} \mathrm{~d} x}
$$

The norm of the error between the modelled and measured lift coefficients gives an indication of the modelling accuracy during an oscillation cycle as a single parameter. In this case, the function $f(x)$ in equation (17) is replaced by

$$
f(\alpha)=\epsilon(\alpha)=c_{l, \text { mod. }}(\alpha)-c_{l, \text { meas. }}(\alpha)
$$

with $c_{l \text {,mod. }}$ the modelled lift coefficient and $c_{l \text {,meas. }}$ the measured values. The integral is with respect to d $\alpha$ and the lower and upper bounds are $a=\alpha_{\text {mean }}-\alpha_{\text {amp }}$ and $b=\alpha_{\text {mean }}+\alpha_{\text {amp }}$, respectively. Since modelled and measured results are only sampled at discrete intervals, in this case at $0.5^{\circ}$ intervals, the error function will yield a vector of local errors. For this vector of local errors, the $L_{2}$ norm, normalised with respect to the number of vector components, is calculated as

$$
\|\epsilon\|_{L_{2}}=\sqrt{\frac{1}{n} \sum_{i=1}^{n} \epsilon_{i}^{2}}
$$

where $\epsilon_{i}=c_{l \text {,mod. }}\left(\alpha_{i}\right)-c_{l \text {,meas. }}\left(\alpha_{i}\right)$, with $\alpha_{1 \ldots n}$ angles first increasing from $\alpha_{\text {mean }}-\alpha_{\text {amp }}$ to $\alpha_{\text {mean }}+\alpha_{\text {amp }}$ and then decreasing back to $\alpha_{\text {mean }}-\alpha_{\text {amp }}$, in steps of $0.5^{\circ}$.

\section{Pitch oscillation of $10^{\circ}$ around a mean angle of attack $\alpha_{\text {mean }}=14^{\circ}$}

The lift coefficient predicted by the dynamic stall models is compared with the measured values, for the lowest oscillation frequency $(k=0.023)$ with the NACA 4415 aerofoil in Figure 4 . All the models seem to predict the magnitude and angle of attack of the maximum lift coefficient fairly accurately. When looking at the $L_{2}$-norm of the difference between measured and calculated lift coefficients, it is clear that the model with the smallest error over an entire pitch cycle is that of Snel with the first-order correction, with the $L_{2}$-norm equal to 0.045 . On the basis of the $L_{2}$-norm, Snel's model with
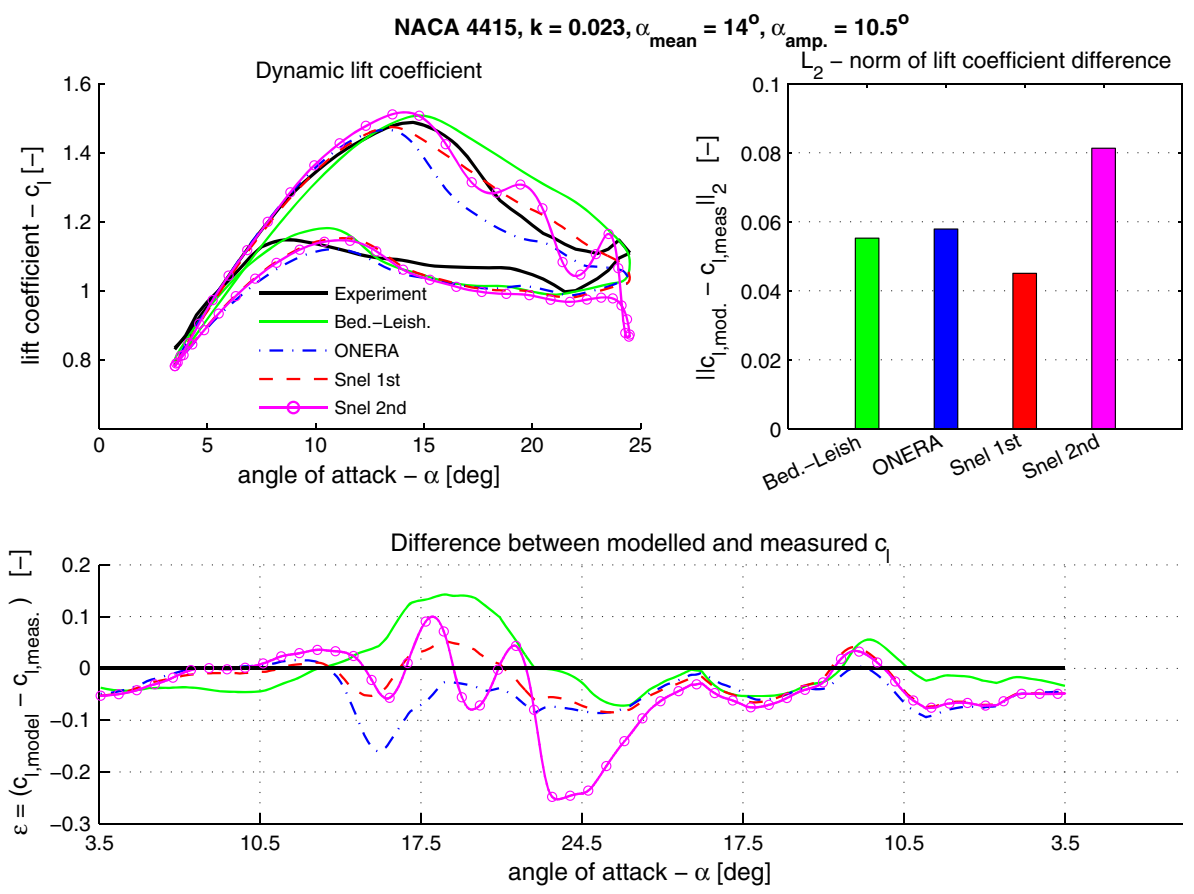

Figure 4. Comparison of measured and modelled results for the NACA 4415 aerofoil; the reduced frequency is $0.023, \alpha_{\text {mean }}=14^{\circ}$, $\alpha_{\text {amp }}=10.5^{\circ}$ and the flow Reynolds number approximately $1.25 \times 10^{6}$. 
the additional non-linear correction performs the worst of all, with the $L_{2}$-norm equal to 0.08 . This is in part due to its modelling of higher frequency dynamics, which is not so significantly present in the measurements and completely disappears because of averaging of multiple cycles (cf. Figure 1 to the averaged plot in Figure 8). This leads to large errors for the last part of the upstroke and the very beginning of the down stroke, as illustrated in the lower part of Figure 4.

For the LS(1)-0142MOD aerofoil, all three models underpredict the maximum lift coefficient, as shown in Figure 5. The Snel method and the Beddoes-Leishman method both predict this maximum lift coefficient at a significantly higher angle of attack; the difference is approximately $2^{\circ}$. Snel's first-order model is again the model with the lowest error norm, with the $L_{2}$-norm equal to 0.045 , very closely followed by the ONERA model $\left(L_{2}\right.$-norm $\left.=0.046\right)$. The model performing worst in this regard is the Beddoes-Leishman model, with the $L_{2}$-norm above 0.06. As shown in the lower plot of Figure 5, the largest differences between the modelled and measured results occur on the upstroke of the oscillation in the stalled region.

Looking at the results of the intermediate oscillation frequency $(k \approx 0.04)$, there are significant differences when compared with the previously discussed results for $k \approx 0.02$. The results are shown for both the S809 aerofoil (see Figure 6) and the LS(1)-0421MOD aerofoil (see Figure 7). Most noticeable is that Snel's non-linear model now consistently has a smaller error norm than the Beddoes-Leishman and ONERA models. For the LS(1)-0421MOD aerofoil, shown in Figure 7, the second-order Snel model even outperforms the first-order Snel model. For higher oscillating frequencies, the higher-order dynamics predicted by the model decrease and the results follow the first-order correction more closely and compares better with the averaged measured values. At this reduced frequency $(k=0.042)$, the Beddoes-Leishman model predicts the largest stall delay of the methods considered with a difference of almost $5^{\circ}$ in maximum lift angle, where the ONERA model under estimates the delay of stall, resulting in errors that are almost twice as large as the error of the Snel models. The lower parts of the figures illustrate that the ONERA model under predicts the lift coefficient for the largest part of the range of angles of attack, with the largest difference in the upstroke.

At the highest measured reduced frequency $k \approx 0.065$, the first-order method of Snel still performs best with respect to the norm of the lift coefficient, with the $L_{2}$-norm $<0.08$, followed by Snel's non-linear model, the Beddoes-Leishman model and finally the ONERA model. The results for the NACA 4415 and S809 aerofoils are shown in Figures 8 and 9 , respectively.

The same trend as for the intermediate reduced frequencies $(k \approx 0.04)$ is observed at this high reduced frequency; the Beddoes-Leishman model predicts the largest stall delay, whereas the ONERA model predicts stall significantly earlier
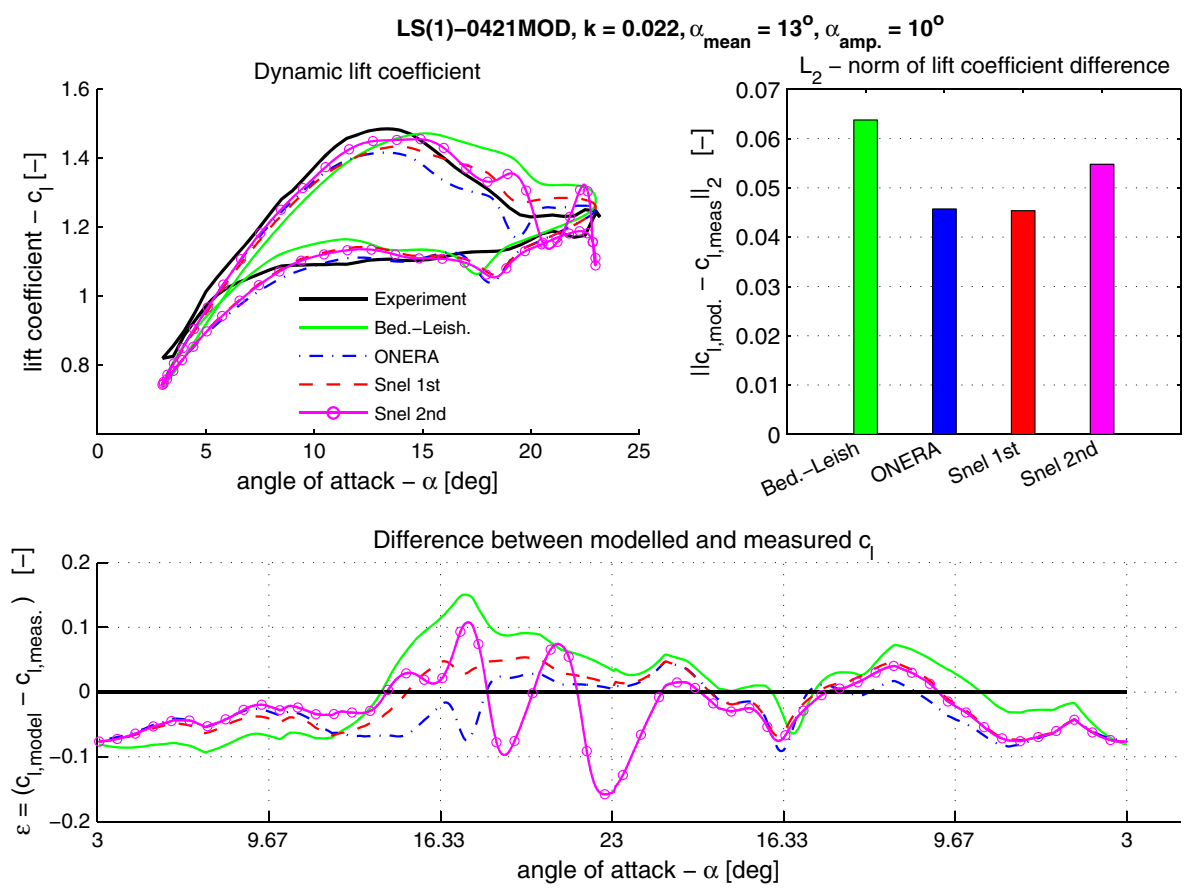

Figure 5. Comparison of measured and modelled results for the LS(1)-0421MOD aerofoil; the reduced frequency is 0.022 , $\alpha_{\text {mean }}=13^{\circ}, \alpha_{\text {amp }}=10^{\circ}$ and the flow Reynolds number approximately $1.25 \times 10^{6}$. 

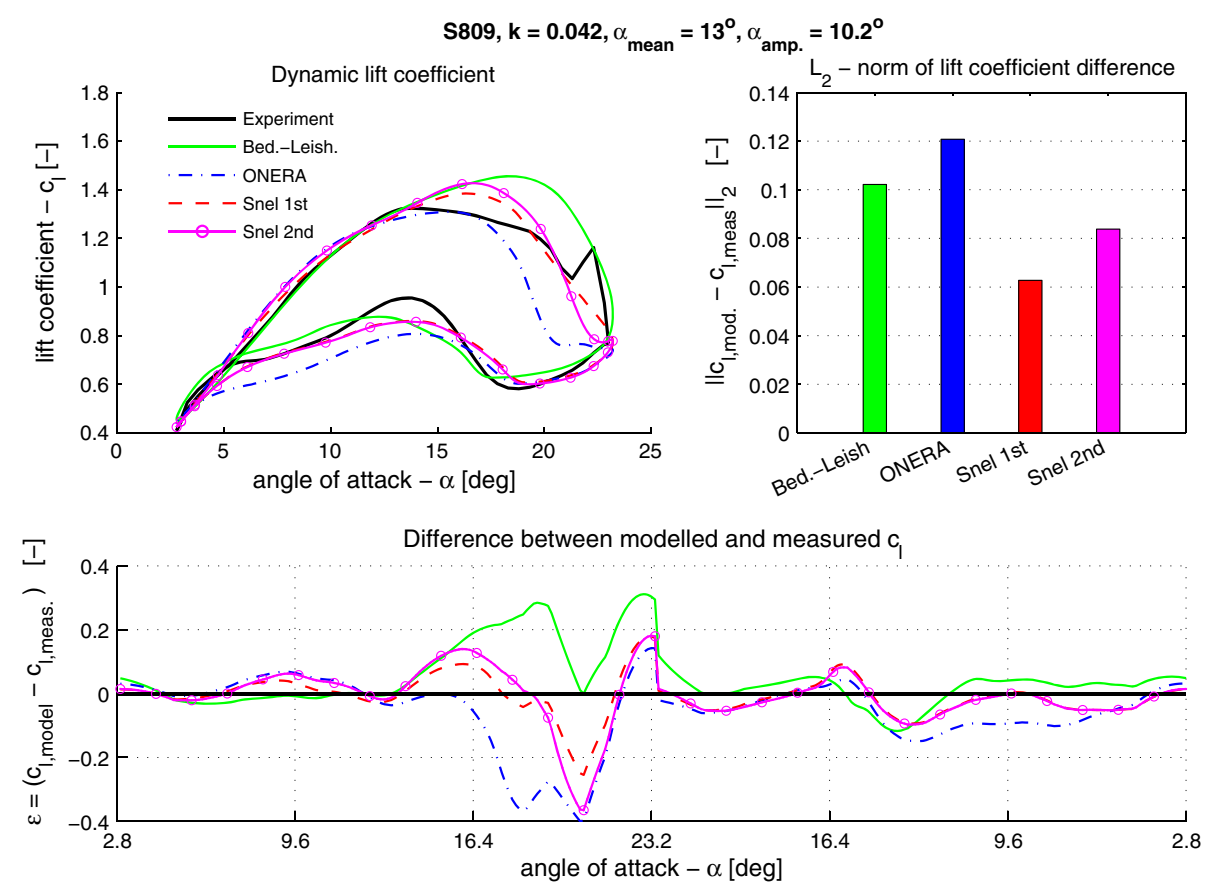

Figure 6. Comparison of measured and modelled results for the $S 809$ aerofoil; the reduced frequency is $0.042, \alpha_{\text {mean }}=13^{\circ}$, $\alpha_{\text {amp }}=10.2^{\circ}$ and the flow Reynolds number approximately $1.25 \times 10^{6}$.
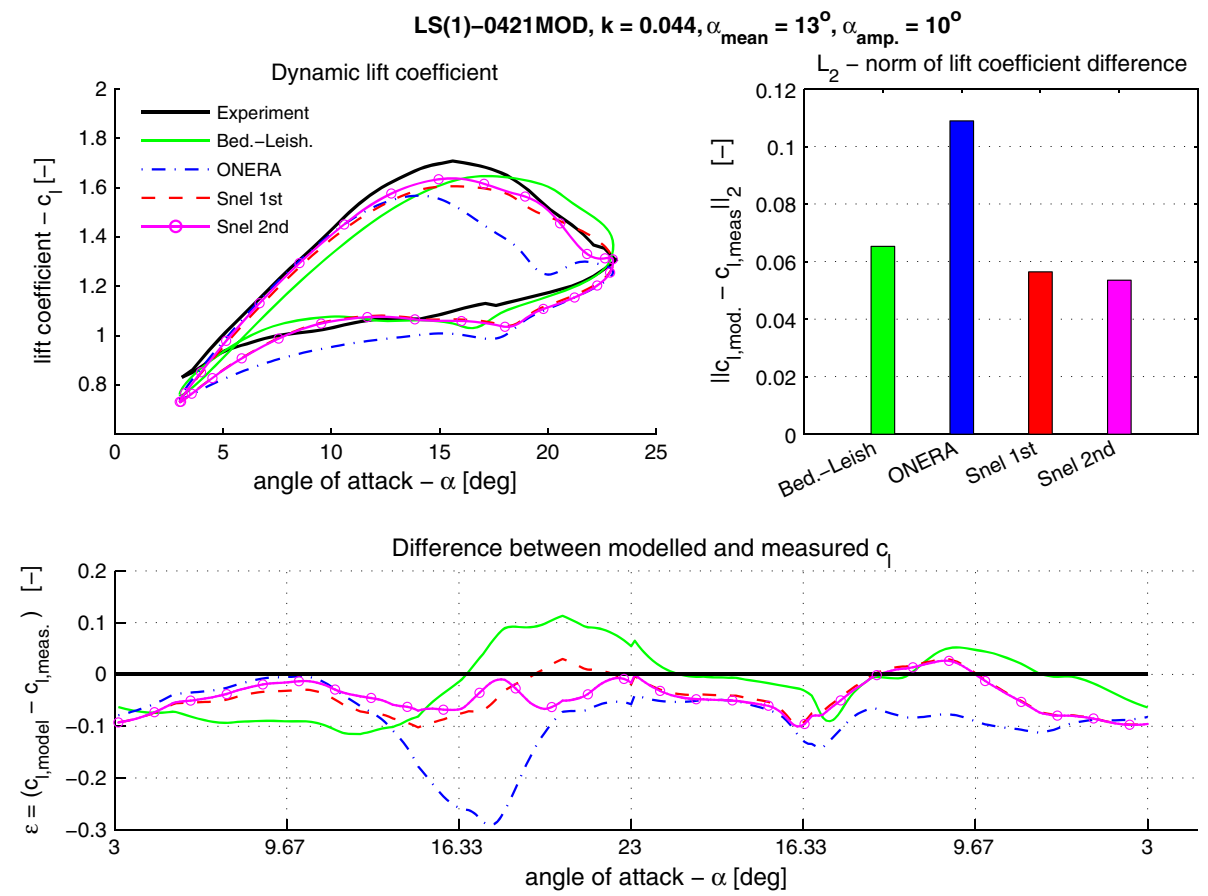

Figure 7. Comparison of measured and modelled results for the LS(1)-0421MOD aerofoil; the reduced frequency is 0.044 , $\alpha_{\text {mean }}=13^{\circ}, \alpha_{\text {amp }}=10^{\circ}$ and the flow Reynolds number approximately $1.25 \times 10^{6}$. 

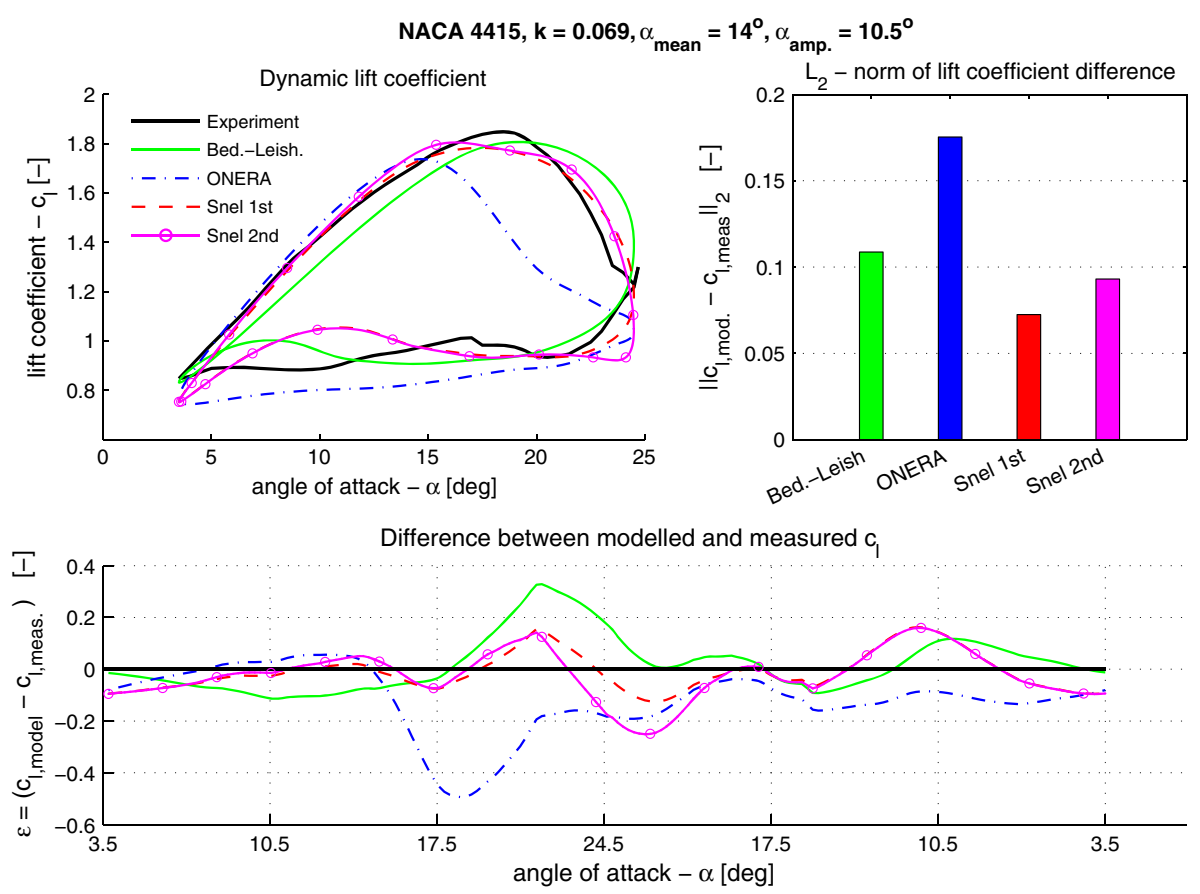

Figure 8. Comparison of measured and modelled results for the NACA 4415 aerofoil; the reduced frequency is $0.069, \alpha_{\text {mean }}=14^{\circ}$, $\alpha_{\mathrm{amp}}=10.5^{\circ}$ and the flow Reynolds number approximately $1.25 \times 10^{6}$.
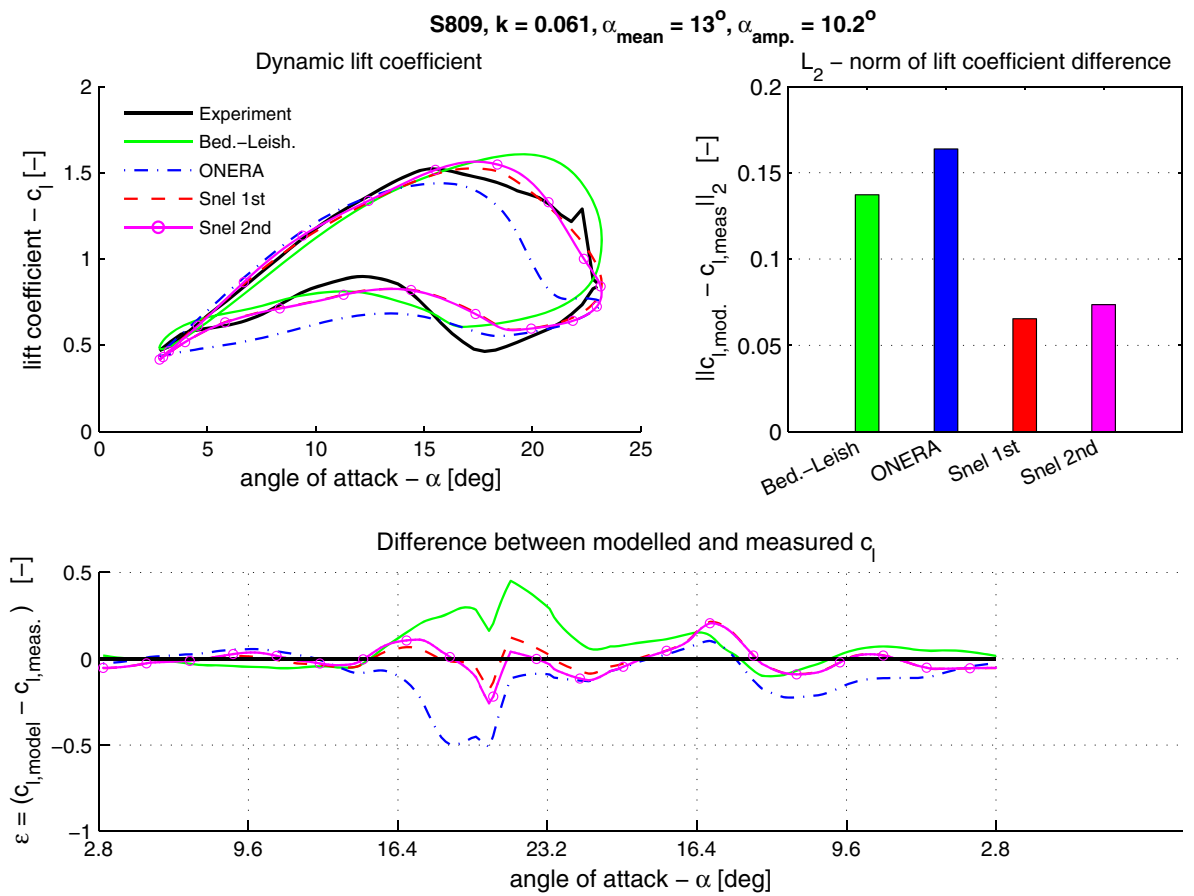

Figure 9. Comparison of measured and modelled results for the $S 809$ aerofoil; the reduced frequency is $0.061, \alpha_{\text {mean }}=13^{\circ}$, $\alpha_{\mathrm{amp}}=10.2^{\circ}$ and the flow Reynolds number approximately $1.25 \times 10^{6}$. 
than in the measurements, with the models of Snel in between these extremes. The significant under prediction occurring in the upstroke for the ONERA model is clearly illustrated in the lower parts of Figures 8 and 9.

For the test cases with a mean angle of attack $\alpha_{\text {mean }}=14^{\circ}$, all the dynamic stall model results show at least the same trends as that of the measurements. In general, it is found that the Beddoes-Leishman model slightly overpredicts both the magnitude and the angle of attack for the maximum lift coefficient. On the other hand, the ONERA model is found to show just the opposite behaviour and slightly underpredicts the mentioned values. The Snel models results usually fall within the extremes set by the other two models. In addition to this, for all the test cases considered, Snel's first-order dynamic stall model resulted in the smallest error norm when compared with the measurements. The second-order correction in the Snel models does not seem to improve the results; in many cases, the results are less accurate while there are additional calculation costs.

The $L_{2}$-norms for all four aerofoils against the reduced frequencies are plotted in Figure 10 . The smallest error obtained in these calculations is an $L_{2}$-norm of 0.045 for the NACA 4415 at a reduced frequency $k=0.023$ using the first-order Snel model. The largest error found is an $L_{2}$-norm of almost 0.18 for the NACA 4415 at the highest reduced frequency $(k=0.069)$ using the ONERA model.

The fact that the models from Snel perform so well in the case of the NACA 4415 and S809 aerofoils at least can to a certain extent be attributed to the fact that the coefficients in the Snel models were derived on the basis of these exact same experimental results. The comparison with the Vertol VR-7 measurements however show a similar trend; again, the Snel models in general outperform the other models.

\section{Pitch oscillation around a mean angle of attack $\alpha_{\text {mean }}=20^{\circ}$}

For the oscillations around a mean angle of attack in the fully stalled regime (for both the static and dynamic case), the shape of the hysteresis is significantly different, especially for the lower reduced frequencies $(k \approx 0.02$ and $k \approx 0.04)$; the shape is much less smooth than the shape observed for the oscillations around $\alpha_{\text {mean }}=14^{\circ}$. The dynamic stall models have difficulty predicting accurate results. This is, however, to be expected, on the basis of the underlying assumptions under which the models were derived. The ONERA model, for instance, is derived on the basis of a linearisation where it is assumed that changes in angle of attack are small and that there is a small difference between the static and dynamic

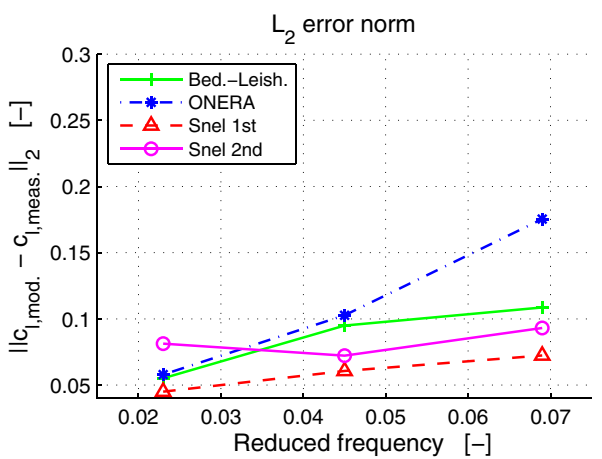

(a) NACA 4415

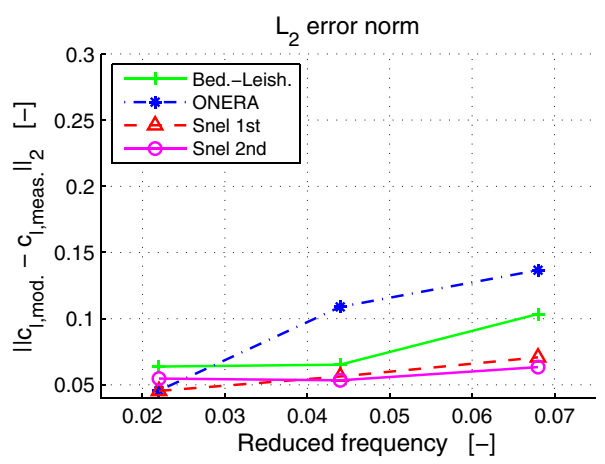

(c) LS(1)-0421MOD

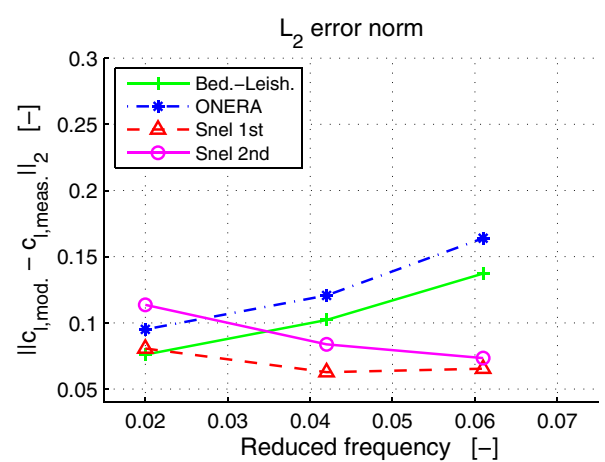

(b) $S 809$

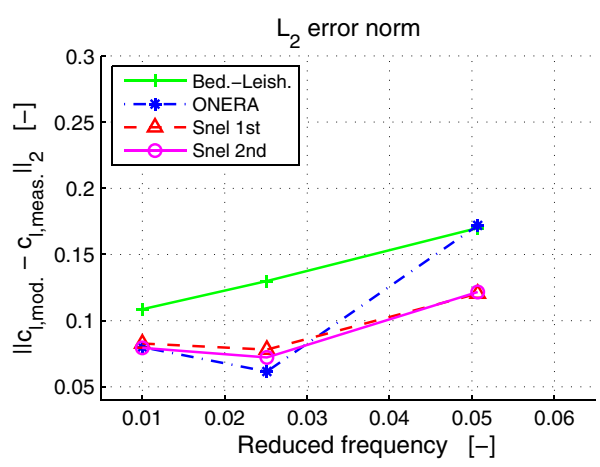

(d) Vertol VR-7

Figure 10. The $L_{2}$ error norm compared with the measured dynamic lift coefficient for the various dynamic stall models as a function of reduced frequency. For the cases considered, $\alpha_{\text {mean }}=15^{\circ}$ and $\alpha_{\text {amp }}=10^{\circ}$. 
lift coefficients, assumptions that are clearly violated. Although some flow physics is involved, Snel's dynamic stall models are largely based on mathematical modelling of stall hysteresis loops around steady stall. The Beddoes-Leishman model is the only model explicitly taking flow separation into account. Therefore, it is not surprising that this is, for the majority of the cases, the model that has the lowest error norm, although the differences with the measurements are still significant.

For both cases of the $5^{\circ}$ and $10^{\circ}$ oscillation amplitudes in the stalled region, the dynamic stall models have difficulty predicting accurate results. This is especially the case for oscillations at the lowest reduced frequency $(k \approx 0.02)$. See e.g. Figure 11, which shows the results for the NACA 4415 aerofoil with $\alpha_{\mathrm{amp}}=10^{\circ}$. Clearly, none of the models closely follows the measured dynamic lift coefficient. In terms of the error norm, the Beddoes-Leishman model performs the best but is clearly not very accurate with a norm that is above 0.1 . The first-order model from Snel performs second best, but the second-order model shows too much higher frequency dynamics on the upstroke, as is clearly illustrated in the lower part of Figure 11.

At higher reduced frequencies, there is some improvement. In Figure 12, the results of the dynamic stall models for the S809 oscillating with an amplitude of $5^{\circ}$ at the intermediate reduced frequency $(k=0.04)$ are shown. The BeddoesLeishman method again shows the smallest error norm of all the methods considered, but there are still substantial deviations from the measured lift coefficient values; the norm is still close to 0.1 . This is a significantly higher norm for the smallest absolute error than was obtained for the vibrations about $\alpha_{\text {mean }}=14^{\circ}$. However, it is a slightly smaller absolute error than was found for the Beddoes-Leishman model for the same oscillating aerofoil about the smaller mean angle of attack (Figure 6).

The last example considered in this comparison is the LS(1)-0421MOD aerofoil undergoing a $10^{\circ}$ pitch oscillation at the highest reduced frequency $(k=0.07)$, for which the results are shown in Figure 13. In this case, the Snel models perform best, where the second-order model has the lowest $L_{2}$-norm equal to 0.11 , followed by the Beddoes-Leishman and ONERA models. The lift hysteresis loop predicted by the ONERA model has a shape similar to the measured hysteresis loop; overall, it represents the shape significantly better than the other models but with an offset in comparison with the measured case, as illustrated in the top left and bottom part of the figure. This results in a relatively high $L_{2}$ norm of 0.14 .

These three dynamic stall models with their current configurations have difficulty in providing accurate results, especially for highly stalled flow conditions. A summary of the results for the fully stalled region is given in Figure 14 for
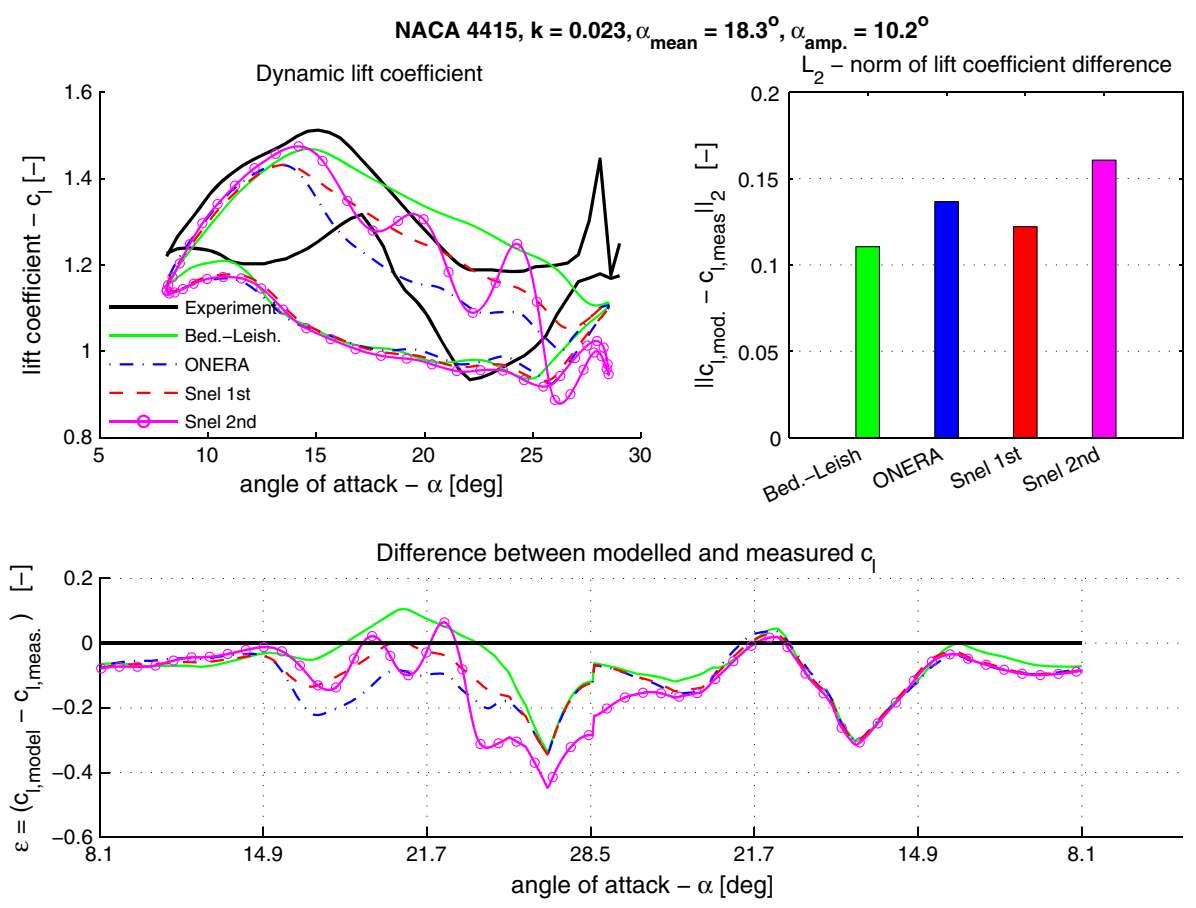

Figure 11. Comparison of measured and modelled results for the NACA 4415 aerofoil; the reduced frequency is $0.023, \alpha_{\text {mean }}=18.3^{\circ}$, $\alpha_{\mathrm{amp}}=10.2^{\circ}$ and the flow Reynolds number approximately $1.25 \times 10^{6}$. 

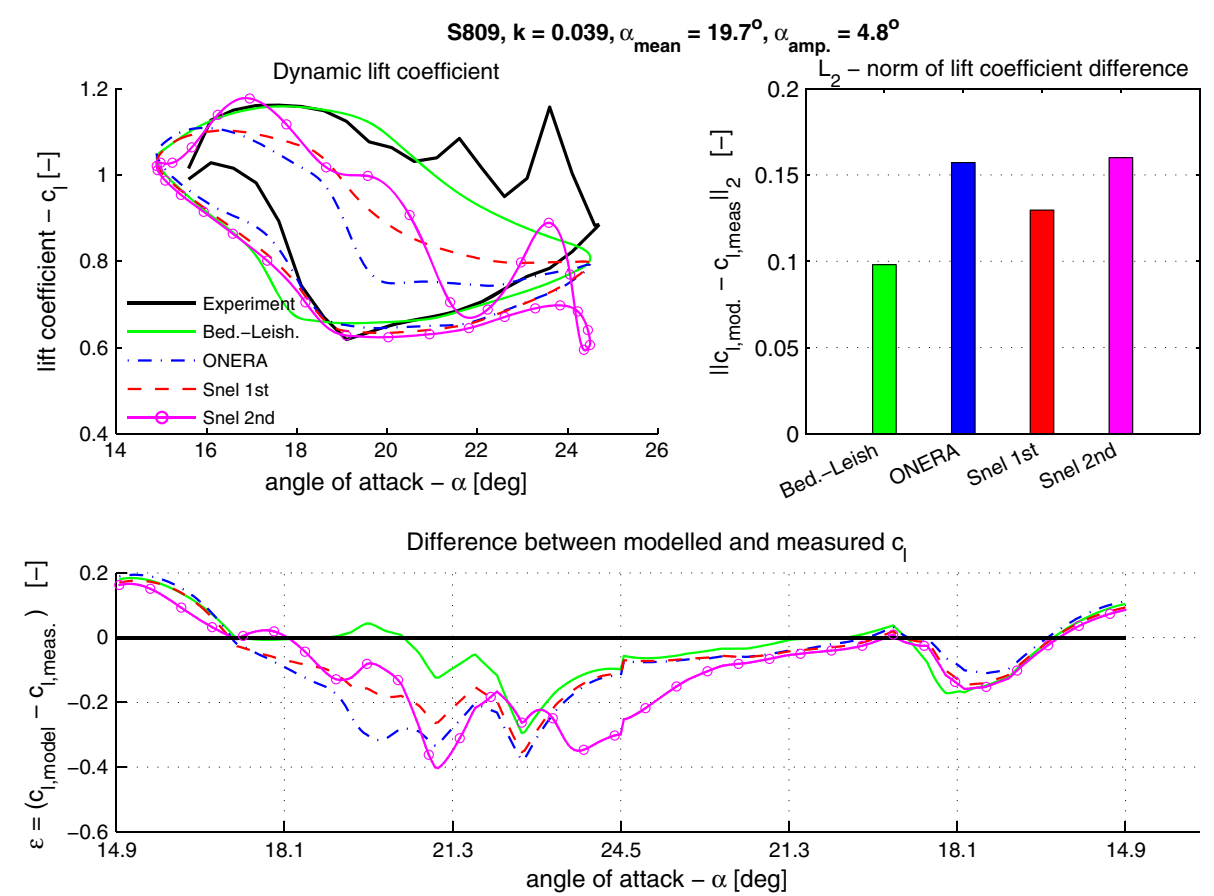

Figure 12. Comparison of measured and modelled results for the $S 809$ aerofoil; the reduced frequency is $0.039, \alpha_{\text {mean }}=19.7^{\circ}$, $\alpha_{\mathrm{amp}}=4.8^{\circ}$ and the flow Reynolds number approximately $1.25 \times 10^{6}$.
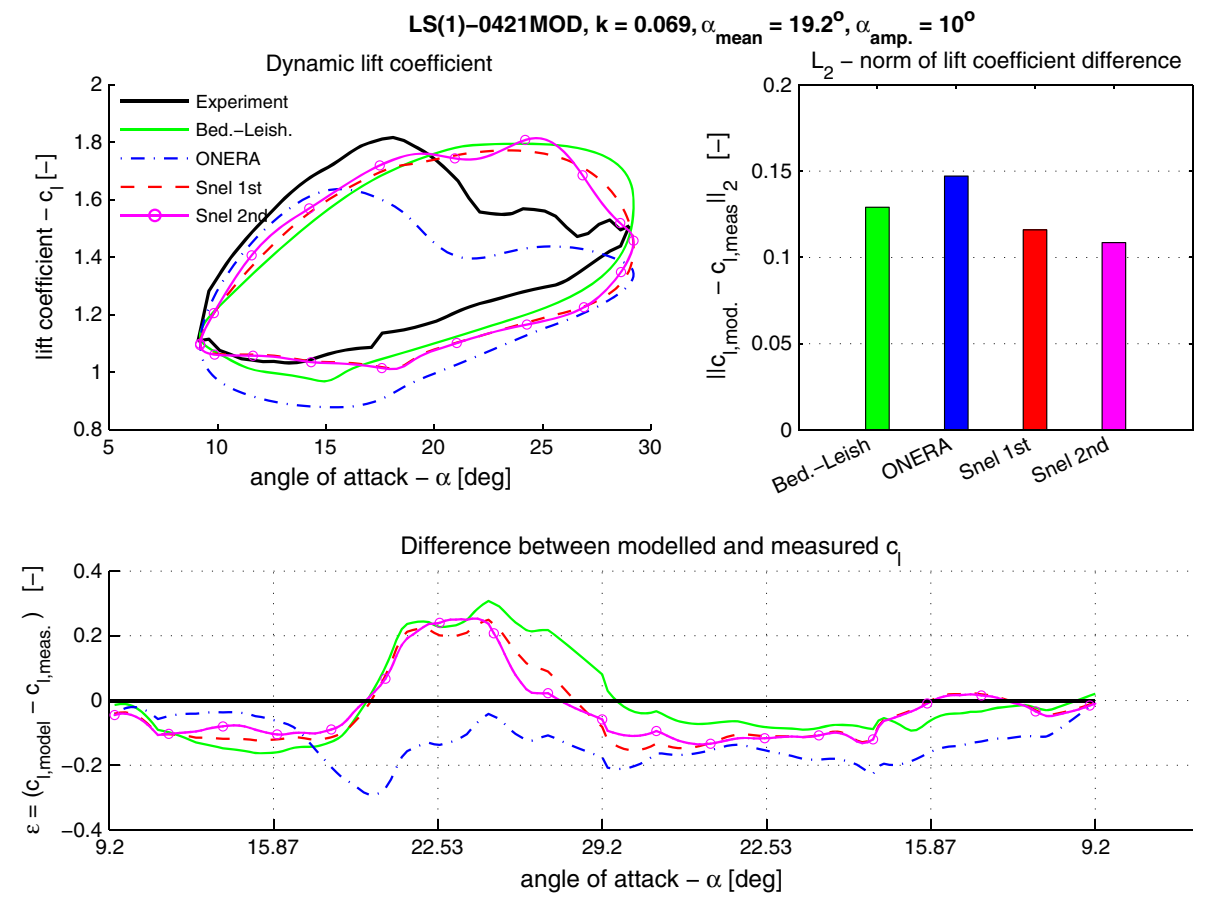

Figure 13. Comparison of measured and modelled results for the LS(1)-0421MOD aerofoil; the reduced frequency is 0.069 , $\alpha_{\text {mean }}=19.2^{\circ}, \alpha_{\mathrm{amp}}=10^{\circ}$ and the flow Reynolds number approximately $1.25 \times 10^{6}$. 


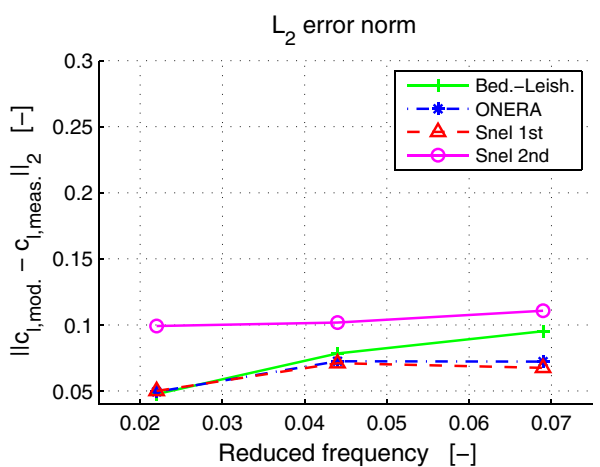

(a) NACA 4415

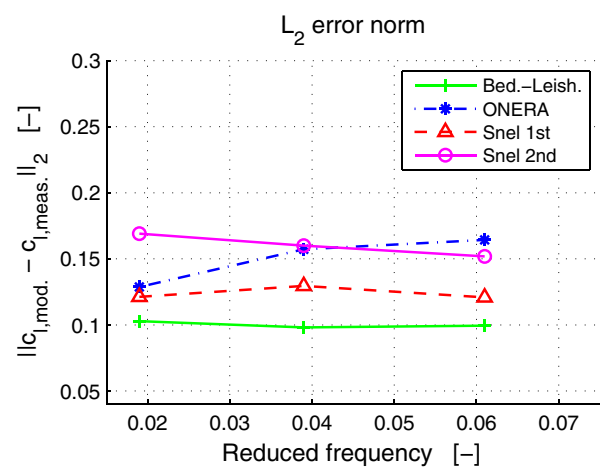

(b) 5809

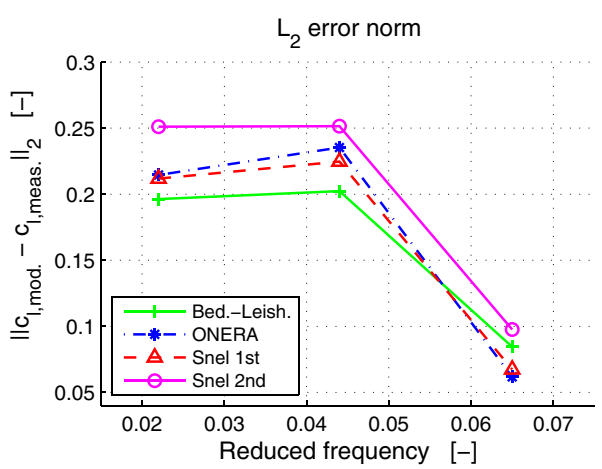

(c) LS(1)-0421MOD

Figure 14. The $L_{2}$ error norm compared with the measured dynamic lift coefficient for the various dynamic stall models as a function of reduced frequency. For the cases considered, $\alpha_{\text {mean }}=20^{\circ}$ and $\alpha_{\text {amp }}=5^{\circ}$.

the cases with an amplitude of $5^{\circ}$ and in Figure 15 for the oscillations with an amplitude of $10^{\circ}$. In these figures, the $L_{2}$-norm values of the different models are shown for the different values of the reduced frequency, for every aerofoil. On average, the smallest absolute error norm is below 0.1, with a few exceptions for higher reduced frequencies and for the LS(1)-0421MOD aerofoil. Judging from these figures, it is difficult to determine a particular trend between the norm of the error and the reduced frequency, with the behaviour varying between different models and for the different aerofoils. However, although the errors are quite large, especially at low reduced frequencies, there seems to be good agreement between the different dynamic stall models on the LS(1)-0421MOD (see Figures 14(c) and 15(c)). In general, the models perform best for the NACA 4415 aerofoil, if the amplitude is $5^{\circ}$. Furthermore, it will be noted that the Beddoes-Leishman or Snel models, especially the first-order model, are regularly the models that show the smallest error for these fully stalled cases.

\section{CONCLUSIONS}

The main conclusion from these comparisons has to be that there is still significant improvement possible in the dynamic stall modelling, especially in the deep stalled regime, which indicates that further research is essential. The largest absolute $L_{2}$ error norm was above 0.25 , e.g. for the LS(1)-0421MOD aerofoil at a reduced frequency $k=0.027, \alpha_{\text {mean }}=20^{\circ}$ and $\alpha_{\mathrm{amp}}=10^{\circ}$. This would have a substantial effect on the outcome of an aeroelastic simulation, for which these dynamic stall models are often used. The lift coefficient varies between 1.3 and 1.6, which renders the absolute error norm above 0.25 as significant. The smallest error norm found was at an acceptable level of 0.04 for the first-order Snel model (NACA 4415 aerofoil, $\alpha_{\text {mean }}=14^{\circ}, k=0.023$ ).

The outcome of the comparison is not really in favour of any of the models. Note that one of the base assumptions was to not use aerofoil specific parameters because of the benefits this will have in aeroelastic simulations, but this will have of course a negative effect on the performance of models that include these parameters such as the ONERA and Beddoes-Leishman models. 


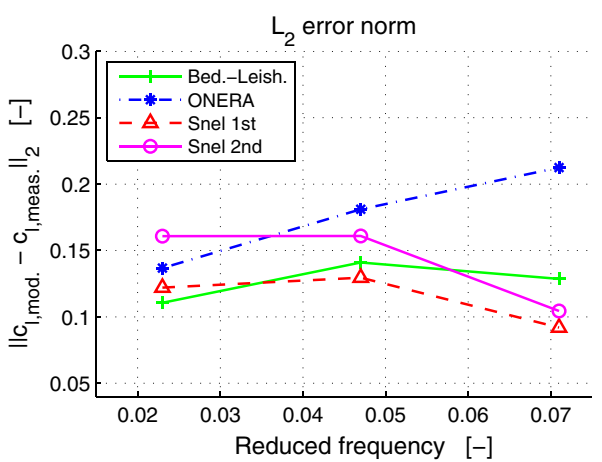

(a) NACA 4415

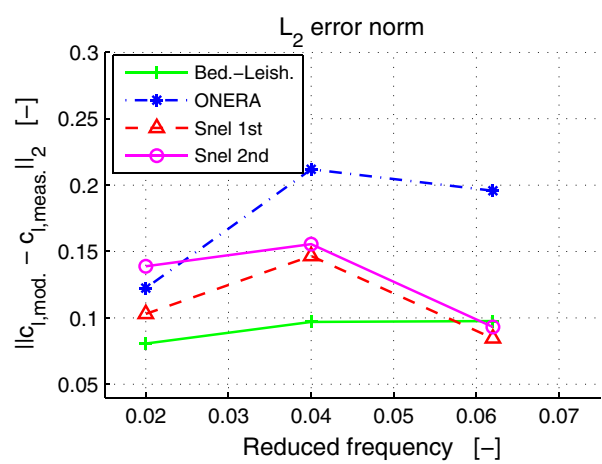

(b) $\$ 809$

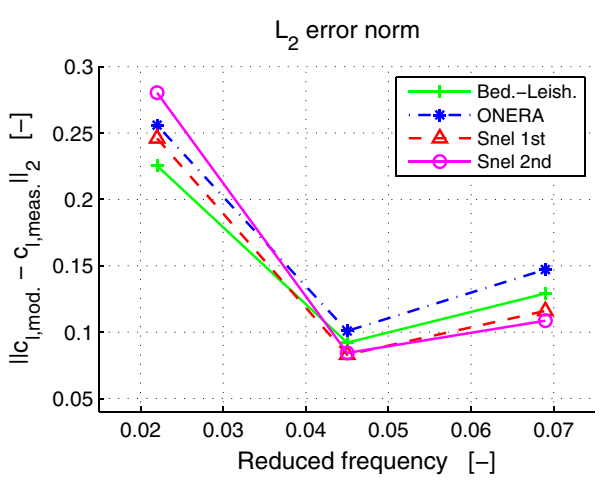

(c) LS(1)-0421MOD

Figure 15. The $L_{2}$ error norm compared with the measured dynamic lift coefficient for the various dynamic stall models as a function of reduced frequency. For the cases considered, $\alpha_{\text {mean }}=20^{\circ}$ and $\alpha_{\text {amp }}=10^{\circ}$.

Furthermore, it can be concluded that the reduced frequency plays a fundamental role in the behaviour of the dynamic lift coefficient. At lower reduced frequencies, the effect of vortex shedding at high angles of attack is more noticeable as a higher frequency component in the lift coefficient, in addition to the response to the forcing frequency. As the reduced frequency increases, this effect becomes less noticeable. This phenomenon is modelled by the second-order model from Snel; however, with the current experimental results, it is difficult to exactly compare this model to the measurements and draw conclusions on the accuracy because of the low measurement frequency.

For oscillations that have a mean angle close to stall and low reduced frequencies, the dynamic stall models give the most accurate results. The difference between measured and calculated lift coefficient in the lift curve does not exceed $10 \%$ of the lift coefficient throughout the loop for the Snel and Beddoes-Leishman models.

If the aerofoil is at an angle of attack that would be stalled in the steady case, the dynamic stall models are especially inaccurate. This is not only due to constraints of the mathematical modelling but also because in the stalled region at these low Reynolds numbers, there is significant unsteadiness in the stalled flow even for a steady angle of attack. This unsteadiness, combined with the mathematical modelling constraints, makes it difficult to accurately predict dynamic stall behaviour in this regime.

It is clear that using a dynamic stall model is essential in aeroelastic simulations, but it is not possible to state that one of the three dynamic stall models is always better than the others. The results also show that the dynamic stall effect will contribute to the differences that show up between the loads that are calculated in the simulations and the loads that are actually measured on the wind turbine. Using any of these models will improve the calculations compared with using steady lift coefficients. To further improve the reliability of the dynamic stall models, each model should be validated and compared with each other for different circumstances, e.g. different aerofoils, reduced frequencies, plunging and pitching motions. Also, the comparison should be extended to include the pitching moments, as for wind turbines that are increasing in size, the torsion deflections become more and more important. These activities could result in an improved reliability for the dynamic stall models and therefore increase the accuracy of the load calculations. 


\section{ACKNOWLEDGEMENTS}

Part of the research has been carried out with financing from the Dutch Ministry of Economic Affairs, in the EOS: Lange Termijn carried out by SenterNovem, assignment number EOS LT 02016, which is gratefully acknowledged.

\section{NOTATION}

\begin{tabular}{|c|c|c|}
\hline$a_{L}$ & Coefficient for ONERA model & {$[-]$} \\
\hline$c$ & The aerofoil chord & {$[\mathrm{m}]$} \\
\hline$c f_{10}$ & Coefficient for constant term in Snel's first-order correction & {$[-]$} \\
\hline$c f_{11}$ & Coefficient for first derivative in Snel's first-order correction & {$[-]$} \\
\hline$c f_{20}$ & Coefficient for constant term in Snel's second-order correction & {$[-]$} \\
\hline$c f_{21}$ & Coefficient for first derivative in Snel's second-order correction & {$[-]$} \\
\hline$c f_{22}$ & Coefficient for second derivative in Snel's second-order correction & {$[-]$} \\
\hline$c_{l, \mathrm{dyn}}$ & Dynamic lift coefficient & {$[-]$} \\
\hline$c_{l, \mathrm{pot}}$ & Potential flow lift coefficient & {$[-]$} \\
\hline$c_{l, \text { stat }}$ & Stationary lift coefficient & {$[-]$} \\
\hline$e_{L}$ & Coefficient for ONERA model & {$[-]$} \\
\hline $\mathrm{ft}_{1}$ & Forcing term in Snel's first-order correction & {$[-]$} \\
\hline$f t_{2}$ & Forcing term for Snel's second-order correction equation & {$[-]$} \\
\hline$k$ & Reduced frequency $k=\omega \tau=\omega c / 2 \mathrm{~V}$ & {$[-]$} \\
\hline$k_{S}$ & Reduced Strouhal frequency & {$[-]$} \\
\hline$r$ & Radial position & {$[\mathrm{m}]$} \\
\hline$R$ & Rotor radius & {$[\mathrm{m}]$} \\
\hline$r_{L}$ & Coefficient for ONERA model & {$[-]$} \\
\hline$P$ & Frequency of rotor rotation & {$[\mathrm{Hz}]$} \\
\hline$s_{L}$ & Coefficient for ONERA model & {$[-]$} \\
\hline$U$ & Undisturbed axial velocity & {$\left[\mathrm{m} \mathrm{s}^{-1}\right]$} \\
\hline$V$ & Effective velocity & {$\left[\mathrm{m} \mathrm{s}^{-1}\right]$} \\
\hline$\alpha$ & Angle of attack defined with respect to chord line & {$[\mathrm{rad}]$} \\
\hline$\alpha_{0}$ & Zero lift angle of attack & [rad] \\
\hline$\Delta c_{l}$ & Lift coefficient difference & {$[-]$} \\
\hline$\Delta c_{l 1}$ & First-order equation lift coefficient correction term & {$[-]$} \\
\hline$\Delta c_{l 2}$ & Second-order equation lift coefficient correction term & {$[-]$} \\
\hline$\lambda_{L}$ & Coefficient for ONERA model & {$[-]$} \\
\hline$\sigma_{L}$ & Coefficient for ONERA model & {$[-]$} \\
\hline$\tau$ & Time constant $\tau=c / 2 \mathrm{~V}$ & {$[\mathrm{~s}]$} \\
\hline$\omega$ & Frequency & {$\left[\mathrm{rad} \mathrm{s}^{-1}\right.$} \\
\hline$\Omega$ & Rotational speed & {$\left[\mathrm{rad} \mathrm{s}^{-1}\right]$} \\
\hline
\end{tabular}

\section{REFERENCES}

1. Schepers J, Snel H. Model experiments in controlled conditions. Technical Report ECN-E-07-042, ECN, Petten, the Netherlands, 2007.

2. Boorsma K. ECN and SIMPACK commence cooperation on wind turbine aero-elastic simulations. SIMPACK News 2009; 2: 12-13.

3. Leishman JG. Challenges in modelling the unsteady aerodynamics of wind turbines. Wind Energy 2002; 5: 85-132.

4. Leishman JG. Principles of Helicopter Aerodynamics, (2nd edn), Cambridge Aerospace Series. Cambridge University Press: Cambridge, 2006.

5. Eggleston DM, Stoddard FS. Wind Turbine Engineering Design. Van Nostrand Reinhold Company: New York, USA, 1987.

6. Sepra DA. Wind Turbine Technology. The American Society of Mechanical Engineers: New York, 1994.

7. Hoffmann MJ, Ramsay RR, Gregorek GM. Effects of grit roughness and pitch oscillations on the NACA 4415 airfoil. Technical Report NREL/TP-442-7815, The Ohio State University/National Renewable Energy Laboratory, 1996.

8. Chaviaropoulos PK. Flap/lead-lag aeroelastic stability of wind turbine blade sections. Wind Energy 1999; 2: 99-112. 
9. Huyer SA, Simms DA, Robinson MC. Unsteady aerodynamics associated with a horizontal-axis wind turbine. AIAA Journal 1996; 34: 1410-1419.

10. Petot D, Arnaud G, Harrison R, Stevens J, Dieterich O, van der Wall BG, Young C, Széchényi E. Stall effects and blade torsion—an evaluation of predictive tools. Journal of the American Helicopter Society 1999; 44: 320-332. DOI: 10.4050/JAHS.44.320. http://dx.doi.org/10.4050/JAHS.44.320.

11. Tan C, Carr L. The AFDD international dynamic stall workshop on correlation of dynamic stall models with 3-D dynamic stall data. Technical Report TM 110375, NASA, USA, 1996.

12. Hoffman MJ, Ramsay RR, Gregorek GM. Unsteady aerodynamic performance of wind turbine airfoils, Proceedings of AWEA Wind Power 94 Conference, Mineapolis, 1994.

13. McAlister KW, Pucci SL, McCroskey WJ, Carr LW. An experimental study of dynamic stall on advanced airfoil sections volume 2. pressure and force data. Technical Report NASA TR 82-A-8, National Aeronautics and Space Administration, 1982.

14. Tran CT, Petot D. Semi-empirical model for the dynamic stall of airfoils in view of the application to the calculation of responses of a helicopter in forward flight. Vertica 1981; 5: 35-53.

15. Dat R, Tran CT. Investigation of the stall flutter of an airfoil with a semi-empirical model of 2-d flow. Vertica 1983; 7: $73-86$.

16. McAlister KW, Lambert O, Petot D. Application of the ONERA model of dynamic stall. Technical Report NASA TR 84-A-3, National Aeronautics and Space Administration, 1984.

17. Peters DA. Towards a unified lift model for use in rotor blade stability analyses, Proceedings of the 40th Annual Forum of the American Helicopter Society, Arlington, VA, May 1984; 525-538.

18. Leishman JG, Beddoes TK. A generalized method for unsteady airfoil behaviour and dynamic stall using the indicial method, 42nd Annual Forum, American Helicopter Society, Washington, D.C., 1986.

19. Leishman JG, Beddoes TS. A semi-empirical model for dynamic stall. Journal of the American Helicopter Society 1986; 34: 3-17.

20. Pierce K, Hansen AC. Prediction of wind turbine rotor loads using the Beddoes-Leishman model for dynamic stall. Journal of Solar Energy Engineering 1995; 117: 200-204.

21. Snel H. Heuristic modelling of dynamic stall characteristics, European Wind Energy conference, Dublin Castle, Ireland, 1997; 429-433.

22. Larsen JW, Nielsen SRK, Krenk S. Dynamic stall model for wind turbine airfoils. Journal of Fluids and Structures 2007; 23: 959-982.

23. Petot D. Differential equation modeling of dynamic stall. La Recherche Aerospatiale (English Edition) 1989; 5: 59-72.

24. Petot D. An investigation of stall on a $4.2 \mathrm{~m}$ diameter experimental rotor. In The Seventh International Workshop on Dynamics and Aeroelastic Modeling of Rotorcraft Systems. U.S. Army Research Office and Washington University in St. Louis: St. Louis, 1997; 1-17, MO (14161997).

25. Brouwer HH. The adaptation of the ONERA model for dynamic stall, for application in the wind-turbine analysis programme PHATAS. In Contract Report NLR CR 90104 L. National Aerospace Laboratory NLR: The Netherlands, 1990.

26. Bierbooms W. A comparison between unsteady aerodynamic models. Journal of Wind Engineering and Industrial Aerodynamics 1992; 39: 23-33.

27. Chaviaropoulos PK. Viscous and aeroelastic effects on wind turbine blades. The Viscel project. Part II: aeroelastic stability investigations. Wind Energy 2003; 6: 387-403. DOI: 10.1002/we.101.

28. Sarkar S, Bijl H. Nonlinear aeroelastic behaviour of an oscillating airfoil during stall-induced vibration. Journal of Fluids and Structures November 2008; 24: 757-777.

29. Beddoes T. Representation of airfoil behaviour. Vertica 1983; 7(2): 183-197.

30. Hansen MH, Guanaa M, Madsen HA. A Beddoes-Leishman type dynamic stall model in state-space and indicial formulations. Technical Report Ris $\phi-R-1354(E N)$, Ris $\varnothing$ National Laboratory, Roskilde, Denmark, June 2004.

31. Gupta S, Leishman JG. Dynamic stall modelling of the s809 aerofoil and comparison with experiments. Wind Energy June 2006; 9: 521-547.

32. Björck A. DYNSTALL: subroutine package with a dynamic stall model. Technical Report FFAP-V-110, FFA: The Aeronautical Research Institute of Sweden, 2001.

33. Björck A. AERFORCE: subroutine package for unsteady blade-element/momentum calculations. Technical Report FFA TN 2000-07, FFA: The Aeronautical Research Institute of Sweden, July 2000. 
34. Truong VK. A 2-D dynamic stall model based on a HOPF bifurcation, 19th European Rotorcraft Forum Proceedings, C23, Cernobbio, Italy, 1993.

35. Montgomerie B. A dynamic stall model called SIMPLE. Technical Report ECN-C-95-060, Netherlands Energy Research Foundation ECN, June 1995.

36. Ramsay RR, Hoffmann MJ, Gregorek GM. Effects of grit roughness and pitch oscillations on the S809 airfoil. Technical Report NREL/TP-442-7817, The Ohio State University/National Renewable Energy Laboratory, December 1995.

37. Ramsay RR, Hoffmann MJ, Gregorek GM. Effects of grit roughness and pitch oscillations on the LS(1)-0421MOD airfoil. Technical Report NREL/TP-422-6473, The Ohio State University/National Renewable Energy Laboratory, December 1995.

38. Sheng W, Galbraith RAM. Coton FN. On the S809 airfoil's unsteady aerodynamic characteristics. Wind Energy 2009; 12: 752-767. DOI: 10.1002/we.331. http://dx.doi.org/10.1002/we.331. 